\title{
Coefficients estimates of some subclasses of analytic functions related with conic domain
}

\begin{abstract}
In this paper, the authors determine the coefficient bounds for functions in certain subclasses of analytic functions related with the conic regions, which are introduced by using the concept of bounded boundary and bounded radius rotations. The effect of certain integral operator on these classes has also been examined.
\end{abstract}

\section{Sarfraz Nawaz Malik, Mohsan Raza, Muhammad Arif and Saqib Hussain}

Let $A$ be the class of functions of the form

$$
f(z)=z+\sum_{n=2}^{\infty} a_{n} z^{n}
$$

which are analytic in the unit disc $E=\{z:|z|<1\}$. Also let $K_{\gamma}$ and $C_{\gamma}^{*}$ denote the well-known classes of close-to-convex and quasi-convex functions of complex order $\gamma(\gamma \neq 0)$ respectively, see for details [2, 12].

Kanas and Wisniowska $[5,6]$ studied the classes of $\beta$-uniformly convex functions denoted by $\beta-U C V$ and the corresponding class of $\beta$ - starlike functions $\beta-S T$ related by the Alexandar type relation. Later Acu [1] considered the class of $\beta$-uniformly close-to-convex functions, denoted by $\beta-U K$ and is defined as:

$\beta-U K=\left\{f(z) \in A: \operatorname{Re}\left(\frac{z f^{\prime}(z)}{g(z)}\right)>\beta\left|\frac{z f^{\prime}(z)}{g(z)}-1\right|, g(z) \in \beta-S T, \quad z \in E\right\}$.

Key Words: Bounded boundary rotations, bounded radius rotations, -uniformly closeto-convex function.

2010 Mathematics Subject Classification: 30C45, 30C50.

Received: February 2013

Accepted: May 2013 
A function $p(z)$, analytic in $E$ with $p(0)=1$, is said to be in the class $P_{k}$, if and only if,

$$
\int_{0}^{2 \pi}|\operatorname{Re} p(z)| d \theta \leq k \pi, k \geq 2 .
$$

This class was introduced by Pinchuk [16]. It is noted that $P_{2}=P$, the well-known class of analytic functions with positive real part. Using the class $P_{k}$, the classes $V_{k}$ of functions with bounded boundary rotations and $R_{k}$ of functions with bounded radius rotations are defined as:

$$
\begin{aligned}
& V_{k}=\left\{f(z) \in A: 1+\frac{z f^{\prime \prime}(z)}{f^{\prime}(z)} \in P_{k}\right\}, \\
& R_{k}=\left\{f(z) \in A: \frac{z f^{\prime}(z)}{f(z)} \in P_{k}\right\} .
\end{aligned}
$$

These classes were studied by Noor $[10,11,15]$ in some details.

Now we define the classes $\beta-U T_{k}(\gamma)$ and $\beta-U T_{k}^{*}(\gamma)$ of analytic functions by using the concepts of bounded boundary rotations and bounded radius rotations as follows.

Definition 1.1. Let $f(z) \in A$. Then $f(z) \in \beta-U T_{k}(\gamma)$, if and only if, there exists a function $g(z) \in R_{k}$ such that

$$
\operatorname{Re}\left(1+\frac{1}{\gamma}\left(\frac{z f^{\prime}(z)}{g(z)}-1\right)\right)>\beta\left|\frac{1}{\gamma}\left(\frac{z f^{\prime}(z)}{g(z)}-1\right)\right|, \quad z \in E
$$

where $\beta \geq 0, k \geq 2$ and $\gamma \in \mathbb{C}-\{0\}$.

Definition 1.2. Let $f(z) \in A$. Then $f(z) \in \beta-U T_{k}^{*}(\gamma)$, if and only if, there exists a function $g(z) \in V_{k}$ such that

$$
\operatorname{Re}\left(1+\frac{1}{\gamma}\left(\frac{\left(z f^{\prime}(z)\right)^{\prime}}{g^{\prime}(z)}-1\right)\right)>\beta\left|\frac{1}{\gamma}\left(\frac{\left(z f^{\prime}(z)\right)^{\prime}}{g^{\prime}(z)}-1\right)\right|, z \in E,
$$

where $\beta \geq 0, k \geq 2$ and $\gamma \in \mathbb{C}-\{0\}$.

It is clear from (1.2) and (1.3) that

$$
f(z) \in \beta-U T_{k}(\gamma) \Leftrightarrow z f^{\prime}(z) \in \beta-U T_{k}^{*}(\gamma) .
$$

It is noticed that by giving specific values to $k, \beta$ and $\gamma$ in the classes $\beta-$ $U T_{k}(\gamma)$ and $\beta-U T_{k}^{*}(\gamma)$, we obtain some well-known subclasses studied by various authors in earlier works, for example, see $[1,2,4,5,6,9,12,13]$. 


\section{Geometric Interpretation}

Geometrically, we see that a function $f(z) \in A$ is in the class $\beta-U T_{k}(\gamma)$ and $\beta-U T_{k}^{*}(\gamma)$, if and only if, the functions $\frac{z f^{\prime}(z)}{g(z)}$ and $\frac{\left(z f^{\prime}(z)\right)^{\prime}}{g^{\prime}(z)}$ respectively take all the values in conic domains $\Omega_{\beta, \gamma}$ with $0<\operatorname{Re} \gamma \leq \beta+1$ such that

$$
\Omega_{\beta, \gamma}=\gamma \Omega_{\beta}+(1-\gamma),
$$

where

$$
\Omega_{\beta}=\left\{u+i v: u>\beta \sqrt{(u-1)^{2}+v^{2}}\right\} .
$$

The domain $\Omega_{\beta, \gamma}$ is elliptic for $\beta>1$, hyperbolic when $0<\beta<1$, parabolic for $\beta=1$ and right half plane when $\beta=0$. This domain has been extensively studied by Noor et.al [14].

The functions which play the role of extremal functions for the conic regions of complex order are given as:

$$
p_{\beta, \gamma}(z)=\left\{\begin{array}{l}
\frac{1+(2 \gamma-1) z}{1-z}, \quad \beta=0, \\
1+\frac{2 \gamma}{\pi^{2}}\left(\log \frac{1+\sqrt{z}}{1-\sqrt{z}}\right)^{2}, \quad \beta=1, \\
1+\frac{2 \gamma}{1-\beta^{2}} \sinh ^{2}\left[\left(\frac{2}{\pi} \arccos \beta\right) \arctan h \sqrt{z}\right], \quad 0<\beta<1, \\
1+\frac{\gamma}{\beta^{2}-1} \sin \left(\frac{\pi}{2 R(t)} \int_{0}^{\frac{u(z)}{\sqrt{t}}} \frac{1}{\sqrt{1-x^{2}} \sqrt{1-(t x)^{2}}} d x\right)+\frac{\gamma}{\beta^{2}-1}, \quad \beta>1,
\end{array}\right.
$$

where $u(z)=\frac{z-\sqrt{t}}{1-\sqrt{t z}}, t \in(0,1), z \in E$ and $z$ is chosen such that $\beta=$ $\cosh \left(\frac{\pi R^{\prime}(t)}{4 R(t)}\right), R(t)$ is the Legendre's complete elliptic integral of the first kind and $R^{\prime}(t)$ is complementary integral of $R(t)$, see $[5,6]$.

We need the following lemmas for our subsequent results.

Lemma 1.3 [16]. Let $p(z) \in P_{k}$. Then $p(z) \in P$ for $|z|<r=\frac{k-\sqrt{k^{2}-4}}{2}$.

Lemma $1.4[8]$. Let $q(z)$ be a convex function in $E$ and let another function $P: E \longrightarrow \mathbb{C}$ with $\operatorname{Re} P(z)>0$. Consider $p(z) \in H(E)$ such that

$$
p(z)+P(z) z p^{\prime}(z) \prec q(z), \quad z \in E .
$$

Then $p(z) \prec q(z), z \in E$.

Main Results

Theorem 2.1. Let $f(z) \in \beta-U T_{k}(\gamma)$ and be given by (1.1). Then

$$
\left|a_{n}\right| \leq\left[\frac{(k)_{n-1}}{n !}+\frac{\left|\delta_{\beta, \gamma}\right|}{n} \sum_{j=1}^{n-1} \frac{(k)_{j-1}}{(j-1) !}\right], \text { for } n \geq 2,
$$


where $\delta_{\beta, \gamma}$ is given by

$$
\delta_{\beta, \gamma}=\left\{\begin{array}{l}
\frac{8 \gamma\left(\cos ^{-1} \beta\right)^{2}}{\pi^{2}\left(1-\beta^{2}\right)}, 0 \leq \beta<1, \\
\frac{8 \gamma}{\pi^{2}}, \quad \beta=1, \\
\frac{\pi^{2} \gamma}{4 \sqrt{t}\left(\beta^{2}-1\right) R^{2}(t)(1+t)}, \quad \beta>1 .
\end{array}\right.
$$

Proof. By definition of $\beta-U T_{k}(\gamma)$

$$
z f^{\prime}(z)=g(z) q(z)
$$

and

$$
q(z) \prec p_{\beta, \gamma}(z),
$$

where $p_{\beta, \gamma}(z)$ is given by $(1.5)$ and $g(z) \in R_{k}$. Let

$$
q(z)=1+\sum_{n=1}^{\infty} d_{n} z^{n} \text { and } g(z)=z+\sum_{n=2}^{\infty} b_{n} z^{n} .
$$

Then from (2.3), we have

$$
z+\sum_{n=2}^{\infty} n a_{n} z^{n}=\left(z+\sum_{n=2}^{\infty} b_{n} z^{n}\right)\left(1+\sum_{n=1}^{\infty} d_{n} z^{n}\right) .
$$

Equating coefficient of $z^{n}$ on both sides, we have

$$
n a_{n}=b_{n}+\sum_{j=1}^{n-1} b_{j} d_{n-j}, \text { for } n \geq 2,
$$

and this implies that

$$
n\left|a_{n}\right|=\left|b_{n}\right|+\sum_{j=1}^{n-1}\left|b_{j}\right|\left|d_{n-j}\right|, \text { for } n \geq 2 .
$$

Since

$$
q(z) \prec p_{\beta, \gamma}(z),
$$

therefore by using the well-known Rogosinski result [17], we have

$$
\left|d_{n-j}\right| \leq\left|\delta_{\beta, \gamma}\right|, \quad j \geq 1,
$$

where $\delta_{\beta, \gamma}$ is given by $(2.2)$. 
Now, using (2.5) and the coefficient estimate $\left|b_{n}\right| \leq \frac{(k)_{n-1}}{(n-1) !}$ of the class $R_{k}$ in $(2.4)$, we have

$$
n\left|a_{n}\right| \leq \frac{(k)_{n-1}}{(n-1) !}+\left|\delta_{\beta, \gamma}\right| \sum_{j=1}^{n-1} \frac{(k)_{j-1}}{(j-1) !}, \text { for } n \geq 2,
$$

which implies that

$$
\left|a_{n}\right| \leq\left[\frac{(k)_{n-1}}{n !}+\frac{\left|\delta_{\beta, \gamma}\right|}{n} \sum_{j=1}^{n-1} \frac{(k)_{j-1}}{(j-1) !}\right] \text {, for } n \geq 2
$$

Hence the proof.

Corollary 2.2. When $\beta=0, k=2$ and $\gamma=1$, then (2.1) reduces to

$$
\left|a_{n}\right| \leq n, \text { for } n \geq 2,
$$

which is the coefficient bound of $f(z) \in K$, the well-known class of close-toconvex functions, introduced by Kaplan, for detail of this class, see [4].

Theorem 2.3. Let $f(z) \in \beta-U T_{k}^{*}(\gamma)$ and be given by (1.1). Then

$$
\left|a_{n}\right| \leq \frac{1}{n}\left[\frac{(k)_{n-1}}{n !}+\frac{\left|\delta_{\beta, \gamma}\right|}{n} \sum_{j=1}^{n-1} \frac{(k)_{j-1}}{(j-1) !}\right], \text { for } n \geq 2,
$$

where $\delta_{\beta, \gamma}$ is given by $(2.2)$.

Proof. By using the relation (1.4) and Theorem 2.3, we obtain the required result.

Corollary 2.4. When $\beta=0, k=2$ and $\gamma=1$, then (2.6) reduces to

$$
\left|a_{n}\right| \leq 1, \text { for } n \geq 2
$$

which is the coefficient bound of $f(z) \in C^{*}$, the well-known class of quasiconvex functions, given by Noor [13].

For the function $f(z) \in A$, we consider the integral operator

$$
I_{a}(f(z))=\frac{a+1}{z^{a}} \int_{0}^{z} t^{a-1} f(t) d t, \quad a \in \mathbb{C} \text { and } \operatorname{Re} a>0 .
$$

This operator $I_{a}$ was introduced by Bernardi [3]. In particular, the operator $I_{1}$ was studied earlier by Libera [7]. 
Now we prove the followings.

Theorem 2.5. Let $f(z) \in \beta-U T_{k}(\gamma)$. Then $I_{a}(f(z)) \in \beta-U T_{k}(\gamma)$ for $|z|<r=\frac{k-\sqrt{k^{2}-4}}{2}$.

Proof. We know that $f(z) \in \beta-U T_{k}(\gamma)$, if and only if, for $g(z) \in R_{k}$, the function $\frac{z f^{\prime}(z)}{g(z)}$ takes all values in the conic domains $\Omega_{\beta, \gamma}$ with $0<\operatorname{Re} \gamma \leq \beta+1$, where $\Omega_{\beta, \gamma}$ is given by (1.5). That is

$$
\frac{z f^{\prime}(z)}{g(z)} \prec p_{\beta, \gamma}(z) .
$$

From (2.9), we have

$$
(1+a) f(z)=z\left(I_{a} f(z)\right)^{\prime}+a I_{a} f(z) .
$$

Also for $g(z) \in R_{k}$,

$$
(1+a) g(z)=z\left(I_{a} g(z)\right)^{\prime}+a I_{a} g(z)
$$

where $I_{a} g(z) \in R_{k}$, see [10]. From (2.10) and (2.11), we obtain

$$
\begin{aligned}
\frac{z f^{\prime}(z)}{g(z)} & =\frac{z\left(z\left(I_{a} f(z)\right)^{\prime}\right)^{\prime}+a z\left(I_{a} f(z)\right)^{\prime}}{z\left(I_{a} g(z)\right)^{\prime}+a I_{a} g(z)} \\
& =\frac{z \frac{\left(z\left(I_{a} f(z)\right)^{\prime}\right)^{\prime}}{I_{a} g(z)}+a \frac{z\left(I_{a} f(z)\right)^{\prime}}{I_{a} g(z)}}{\frac{z\left(I_{a} g(z)\right)^{\prime}}{I_{a} g(z)}+a} .
\end{aligned}
$$

Now let $\frac{z\left(I_{a} f(z)\right)^{\prime}}{I_{a} g(z)}=p(z)$ and $\frac{z\left(I_{a} g(z)\right)^{\prime}}{I_{a} g(z)}=p_{1}(z)$. Then we have

$$
z\left(I_{a} f(z)\right)^{\prime}=p(z)\left(I_{a} g(z)\right)
$$

Differentiating the above equality with respect to $z$, we get

$$
\left(z\left(I_{a} f(z)\right)^{\prime}\right)^{\prime}=p^{\prime}(z)\left(I_{a} g(z)\right)+p(z)\left(I_{a} g(z)\right)^{\prime} .
$$

This implies that

$$
\frac{z\left(z\left(I_{a} f(z)\right)^{\prime}\right)^{\prime}}{I_{a} g(z)}=z p^{\prime}(z)+p(z) p_{1}(z) .
$$

Using this in (2.12), we have

$$
\frac{z f^{\prime}(z)}{g(z)}=p(z)+\frac{z p^{\prime}(z)}{p_{1}(z)+a} .
$$


From (2.10) and (2.13), we have

$$
p(z)+\frac{z p^{\prime}(z)}{p_{1}(z)+a} \prec p_{\beta, \gamma}(z) .
$$

Since $\operatorname{Re}\left[p_{1}(z)+a\right]>0$ for $|z|<r=\frac{k-\sqrt{k^{2}-4}}{2}$, by Lemma 1.3. So by using Lemma 1.4, we have

$$
p(z) \prec p_{\beta, \gamma}(z) .
$$

Therefore $I_{a}(f(z)) \in \beta-U T_{k}(\gamma)$.

Theorem 2.6. Let $f(z) \in \beta-U T_{k}^{*}(\gamma)$. Then $I_{a}(f(z)) \in \beta-U T_{k}^{*}(\gamma)$ for $|z|<r=\frac{k-\sqrt{k^{2}-4}}{2}$.

Proof. Using the same procedure as given in Theorem 2.5, we can easily obtain the required result.

Acknowledgement. The authors are thankful to referee for his valuable suggestions.

\section{References}

[1] M. Acu, On a subclass of $n$-uniformly close-to-convex functions, Gen. Math., 14(2006) 55-64.

[2] H. S. Al-Amiri and T. S Fernando, On close-to-convex functions of complex order, Int. J. Math. Math. Sci., 13(1990) $321-330$.

[3] S. D. Bernardi, Convex and Starlike Univalent Functions, Trans. Amer. Math. Soci., 135(1969) $429-446$.

[4] A. W. Goodman, Univalent functions, Vol. I, II, Mariner Publishing Company, Tempa, Florida, U. S. A, 1983.

[5] S. Kanas and A. Wisniowska, Conic regions and k-uniform convexity, J. Comput. Appl. Math., 105(1999) $327-336$.

[6] S. Kanas and A. Wisniowska, Conic domains and starlike functions, Rev. Roumaine Math. Pures Appl., 45(2000) 647 - 657.

[7] R. J. Libra, Some Classes of Regular Univalent Functions, Proc. Amer. Math. Soc., 16(1965) $755-758$.

[8] S. S. Miller and P. T. Mocanu, Univalent solution of Briot-Bouquet differential equations, J. Differential Equations, 56(1985), 297 - 308.

[9] K. I. Noor, On a generalization of close-to-convexity, Int. J. Math. Math. Sci., 6(2)(1983) $327-334$. 
[10] K. I. Noor, On a generalization of uniformly convex and related functions, Comput. Math. Appl., 61 (1) (2011) $117-125$.

[11] K. I. Noor, On some subclasses of functions with bounded boundary and bounded radius rotation, Pan Amer. Math. J., 6(1996) 75 - 81.

[12] K. I. Noor, Quasi-convex functions of complex order, Pan Amer. Math. J., 3(2)(1993) $81-90$.

[13] K. I. Noor and D. K. Thomas, Quasi-convex univalent functions, Int. J. Math. Math. Sci., 3(1980)255 - 266.

[14] K. I. Noor, M. Arif and W. Ul-Haq, On k-uniformly close-to-convex functions of complex order, Appl. Math. Comput., 215(2)(2009) 629 - 635.

[15] K. I. Noor, W. Ul-Haq, M. Arif and S. Mustafa, On Bounded Boundary and Bounded Radius Rotations, J. Inequ. Appl., (2009) articles ID 813687, 12 pages.

[16] B. Pinchuk, Functions with bounded boundary rotation, Isr. J. Math., $10(1971) 7-16$.

[17] W. Rogosinski, On the coefficients of subordinate functions, Proc. London Math. Soc., 48 (1943) $48-82$.

Sarfraz Nawaz Malik,

Department of Mathematics,

Govt. College University,

Faisalabad, Pakistan.

Email: snmalik110@yahoo.com

Mohsan Raza,

Department of Mathematics,

Govt. College University,

Faisalabad, Pakistan.

Email: mohsan976@yahoo.com

Muhammad Arif,

Department of Mathematics,

Abdul Wali Khan University Mardan, Pakistan.

Email: marifmaths@yahoo.com

Saqib Hussain,

Department of Mathematics,

COMSATS Institute of Information Technology,

Abbottabad, Pakistan.

Email: saqib_math@yahoo.com 